\title{
Precise determination of optical properties of pentacene thin films grown on various substrates: Gauss-Lorentz model with effective medium approach
}

\author{
S.-H. Han • S. Yoo • B. Kippelen • D. Levi
}

Received: 6 October 2010 / Revised version: 8 December 2010 / Published online: 18 February 2011

(C) The Author(s) 2011. This article is published with open access at Springerlink.com

\begin{abstract}
Spectroscopic ellipsometry measurements are performed on thin pentacene films grown on glass, $\mathrm{SiO}_{2}$, and $n$-Si substrates. The Gauss-Lorentz oscillator model is shown to be effective in modeling the $\pi-\pi^{*}$ transitions found in organic compounds. The effective medium approximation that considers the surface roughness of the films, which can be significant in case of pentacene, is also shown to be a key factor in precisely determining their dielectric functions. The proposed method reveals that there are some quantitative differences in the optical properties of the pentacene films prepared on different substrates.
\end{abstract}

\section{Introduction}

Pentacene $\left(\mathrm{C}_{22} \mathrm{H}_{14}\right)$ is an organic material attracting much attention as a hole transport layer in various electronic devices. Thin pentacene films have been widely used in organic field-effect transistors (OFETs) [1] mainly due to

S.-H. Han $(\varangle) \cdot$ D. Levi

National Renewable Energy Laboratory, 1617 Cole Boulevard, Golden, CO 80401-3393, USA

e-mail: sungho.han@1ge.edu

S.-H. Han

LG Electronics Institute of Technology, 16 Woomyeon-Dong, Seocho-Gu, Seoul 137-724, Korea

S. Yoo $\cdot$ B. Kippelen

Center for Organic Photonics and Electronics, School

of Electrical and Computer Engineering, Georgia Institute

of Technology, Atlanta, GA 30332-0250, USA

S. Yoo

Department of Electrical Engineering, Korea Advanced Institute of Science and Technology, Daejon, Korea the polycrystalline nature leading to high hole mobilities $[2,3]$. Thin pentacene films have also been used as photoactive hole transport layers in organic photovoltaic (OPV) cells [4, 5], photodiodes [6], and phototransistors [7, 8]. In contrast to OFETs that presumably operate in dark conditions, the complex dielectric function $\tilde{\epsilon}(\omega)=\epsilon_{1}(\omega)+i \epsilon_{2}(\omega)$ serves as an important set of information in understanding and optimizing organic photonic devices. For instance, the structure of multilayer thin-film OPV cells can be optimized to yield a large photocurrent based on the optical model that is a function of the thickness and complex dielectric functions of each participating layer $[9,10]$.

In such respect, there have been efforts to obtain the complex dielectric functions of thin pentacene films in the visible region (1.5-2.2 eV) [11] as well as in the far-infrared region [12]. We note that pentacene thin films typically have a surface roughness the degree of which can vary significantly depending on the preparation condition. The presence of a surface roughness can influence the ellipsometric data, and thus one could over- or underestimate the true optical constants of a given thin film unless its effect is correctly taken into account. To make things more complicated, the different preparation conditions for pentacene films can result in a different polymorph, which can impact their electronic and thus optical properties. Therefore, it is crucial to establish an effective and robust way to extract the optical properties of pentacene, or organic material in general, regardless of preparation conditions. Here, we show that a combination of a Gauss-Lorentz oscillator model and effective-medium approximation (EMA) can precisely determine the dielectric functions of thin pentacene films deposited on some representative substrates that may be of interest for optoelectronic device applications $[4,6,8]$. It is revealed that the dielectric functions of those pentacene films on different sub- 


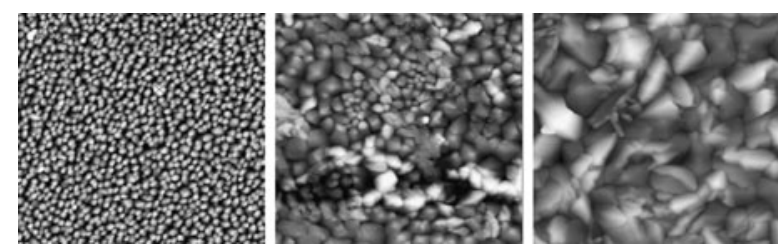

Fig. 1 Two-dimensional atomic force microscopy (AFM) images of thin pentacene films on glass, $\mathrm{SiO}_{2}$, and $n$-Si from the left. The scan size of each image is $5 \times 5 \mu \mathrm{m}^{2}$

strates have significant difference. The possible origin of the substrate-dependent optical properties is also discussed.

\section{Experimental details}

\subsection{Film growth and characterization}

Nominally $100 \mathrm{~nm}$ thick pentacene films were deposited on a microscope slide (later on, referred to as glass), thermally grown $\mathrm{SiO}_{2}$ on $n-\mathrm{Si}$ (later on, referred to as $\mathrm{SiO}_{2}$ ), and $n-\mathrm{Si}$ substrates by vacuum sublimation at a base pressure of less than $10^{-7}$ Torr as described previously [4]. Substrates were held at room temperature during the depositions.

Two-dimensional atomic force microscopy (AFM) images of thin pentacene films prepared on the glass; $\mathrm{SiO}_{2}$ and $n$-Si substrates are shown in Fig. 1. It shows the relatively high crystallinity with grains of a few hundred nanometers for thin pentacene films on $\mathrm{SiO}_{2}$ and $n$-Si and the rather poor crystallinity with smaller grains for pentacene films on glass. X-ray diffraction results show a consistent trend, that is, large peaks for films on $\mathrm{SiO}_{2}$ and $n-\mathrm{Si}$, and small peaks for films on glass (see Fig. 2). The fact that films on glass substrates has a poor crystallinity is not surprising considering the typical roughness of a microscope slide glass. If a polished glass substrate is used instead, it is likely that the crystallinity similar to that of the films grown on $\mathrm{SiO}_{2}$ is obtained. The main purpose of this work is not to grow highly crystalline pentacene films but to obtain the optical properties of those with varying degree of morphologies and crystallinity that can be found in various kinds of experiment.

\subsection{Variable angle spectroscopic ellipsometry}

Note in AFM images that surface roughness $R_{\mathrm{rms}}$ of the pentacene films holds a significant portion of the total thickness (see Fig. 3 and Table 1). In spectroscopic ellipsometry, such roughness can be conveniently modeled using the EMA [13]. With this approximation, we used a multilayer model in which the layers are a substrate layer, a neat thin pentacene film layer, and a surface roughness layer. In addition, noting the previous report that the use of only Lorentzian or Gaussian model tends to provide a rather poor
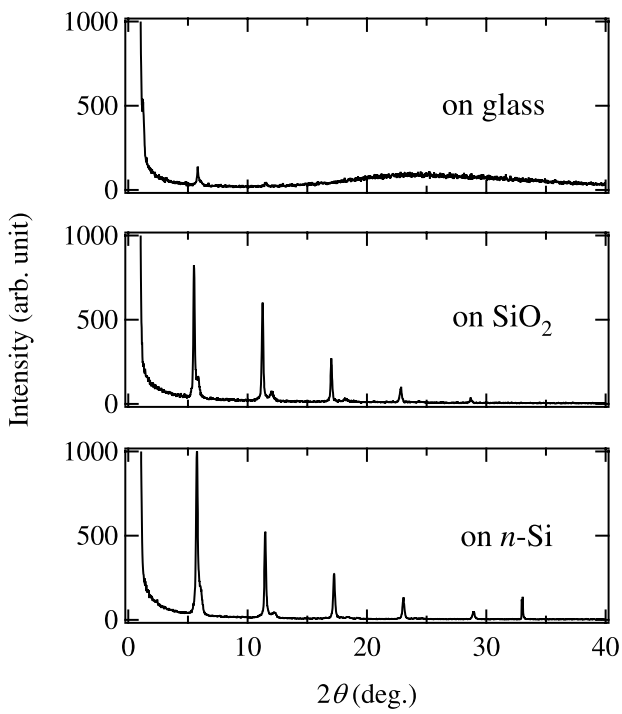

Fig. 2 XRD spectra of thin pentacene films on glass, $\mathrm{SiO}_{2}$, and $n-\mathrm{Si}$ from the top

Fig. 3 Schematic diagram of each sample used in the analysis. Model layers are composed of rough surface roughness layer, neat pentacene film layer, and a substrate

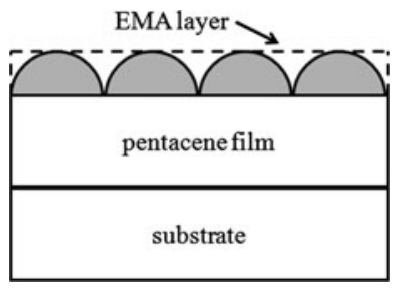

Table 1 Surface roughness analysis using AFM and roughness parameter and $\epsilon_{1}$ offset $\left(\epsilon_{1}(\infty)=\epsilon_{\infty}\right)$ used in ellipsometric analysis. $R_{\mathrm{rms}}$ is the experimental root-mean-square thickness and $R_{\mathrm{SE}}$ was determined from the ellipsometry measurement

\begin{tabular}{lcc}
\hline Substrate & $R_{\mathrm{rms}}$ & $R_{\mathrm{SE}}$ \\
\hline glass & 11.9 & 11.2 \\
$\mathrm{SiO}_{2}$ & 19.5 & 19.9 \\
$n-\mathrm{Si}$ & 33.9 & 27.2 \\
\hline
\end{tabular}

fit to experimental data of thin organic films [14, 15], we employed the so-called Gauss-Lorentz oscillator model similar to the model developed by Kim and Garland for inorganic semiconductors [16]. In this model, contributions from both models are combined to explain the net lineshapes that result from various broadening mechanisms.

\section{Theory}

\subsection{Definitions of basic ellipsometric terms}

Spectroscopic ellipsometry uses the change in the state of polarization of light upon the reflection off the sample. This 
change is related to the dielectric functions through the measurements of $\Psi$ and $\Delta$, where $\tan (\Psi)$ is the amplitude ratio of complex reflection coefficients and $\Delta$ is the phase change in $p$ and $s$ direction. The complex quantity $\tilde{\rho}$ is defined by

$\tilde{\rho} \equiv \frac{\tilde{r}_{p}}{\tilde{r}_{s}}=\frac{\left|\tilde{r}_{p}\right|}{\left|\tilde{r}_{s}\right|} e^{i\left(\delta_{p}-\delta_{s}\right)} \equiv \tan (\Psi) \exp (i \Delta)$,

where $\tilde{r}_{p}$ and $\tilde{r}_{s}\left(\delta_{p}\right.$ and $\left.\delta_{s}\right)$ are the complex reflection coefficients (phase changes) for light polarized parallel $(p)$ and perpendicular $(s)$ to the plane of incidence. More details on the spectroscopic ellipsometry is discussed elsewhere [13].

\subsection{Model dielectric function: Gauss-Lorentz model}

The dielectric function in the interband transitions of a semiconducting material is related to their electronic structure. The relationship between $\epsilon_{2}(\omega)$ and electronic structure is given by [17]

$\epsilon_{2}(\omega)=\frac{4 e^{2}}{\pi \mu^{2} \omega^{2}} \int d \mathbf{k}\left|P_{c v}(\mathbf{k})\right|^{2} \delta\left[E_{c}(\mathbf{k})-E_{v}(\mathbf{k})-\hbar \omega\right]$,

where $\mu$ is the mass, the Dirac $\delta$ function represents the joint density of states between the conduction and valence bands with the difference in energy $\hbar \omega, P_{c v}(\mathbf{k})$ is the matrix element between conduction and valence band states, and the integral is performed over the first Brillouin zone. $P_{c v}(\mathbf{k})$ is defined as

$P_{c v}(\mathbf{k})=\frac{1}{\Delta}\left|\int u_{c \mathbf{k}}^{*} p_{\mu} u_{v \mathbf{k}} d \mathbf{r}\right|$,

where $\Delta$ is the volume of the unit cell, $p_{\mu}$ is the component of the moment operator, and $u_{c \mathbf{k}}$ and $u_{v \mathbf{k}}$ is the wave function at conduction and valence band at $\mathbf{k}$.

Kim and Garland model states that following line shapes [16]

$$
\begin{aligned}
\tilde{\epsilon}_{n}(\omega)= & +i \frac{8 \pi \hbar^{2} e^{2}}{m^{2}} \sum_{c, v} \int J_{c v}(E) d E\left(\frac{P_{c v}(E)}{E}\right)^{2} \\
& \times\left(\int_{0}^{\infty} e^{i\left(\hbar \omega-E_{n}+i \gamma_{n}(s)\right) s} d s\right. \\
& \left.-\int_{0}^{\infty} e^{i\left(\hbar \omega+E_{n}+i \gamma_{n}(s)\right) s} d s\right)
\end{aligned}
$$

where $J_{c v}(E)$ is the joint density of states between a pair of bands and $P_{c v}(E)$ is the weighted-average matrix element of the momentum operator. In order to obtain a usable formula for $\tilde{\epsilon}_{n}(\omega)$, they expand $\gamma_{n}(s)$ as a power series in $s=t / \hbar$, where $t$ is time, and express it up to a linear term:

$\gamma_{n}(s)=\Gamma_{n}+2 \sigma_{n}^{2} s$.
If there is no effect of line broadening $\left(\gamma_{n}(s) \rightarrow 0\right)$ for $n$th oscillator $\tilde{\epsilon}_{n}(\omega)$, (4) is reduced to the typical optical transition formula

$$
\begin{aligned}
\epsilon_{2}(\omega) & =\Im(\tilde{\epsilon}(\omega)) \\
& =\frac{8 \pi^{2} \hbar^{2} e^{2}}{m^{2}} \sum_{c, v} \int J_{c, v}(E) d E\left(\frac{P_{c v}(E)}{E}\right)^{2} \delta(\hbar \omega-E) \\
& =8\left(\frac{\pi e}{m \omega}\right)^{2} \sum_{c, v}\left[P_{c v}(\hbar \omega)\right]^{2} J_{c v}(\hbar \omega) .
\end{aligned}
$$

If the joint density of state function $J_{c v}(E)$ is replaced by a Dirac $\delta$ function $\delta(E-\hbar \omega)$ in the integral, (4) yields the results

$$
\begin{aligned}
\tilde{\epsilon}_{n}(\omega)= & +i \frac{8 \pi \hbar^{2} e^{2}}{m^{2}} \sum_{c, v}\left(\frac{P_{c v}(\hbar \omega)}{\hbar \omega}\right)^{2} \\
& \times\left(\int_{0}^{\infty} e^{i\left(\hbar \omega-E_{n}+i \gamma_{n}(s)\right) s} d s\right. \\
& \left.-\int_{0}^{\infty} e^{i\left(\hbar \omega+E_{n}+i \gamma_{n}(s)\right) s} d s\right),
\end{aligned}
$$

If we disregard the $\epsilon_{1}$ offset 1 , which will be considered in the actual fitting process and is not usually 1 , and set $8 \pi e^{2} \sum_{c, v}\left[P_{c v}(\hbar \omega)\right]^{2} / m^{2} \omega^{2} \equiv A_{n}$, then the resulting $n$th Gauss-Lorentz oscillator model yields the result

$$
\begin{aligned}
\tilde{\epsilon}_{n}(\omega)= & i A_{n}\left[\int_{0}^{\infty} e^{i\left(\hbar \omega-E_{n}+i \gamma_{n}(s)\right) s} d s\right. \\
& \left.-\int_{0}^{\infty} e^{i\left(\hbar \omega+E_{n}+i \gamma_{n}(s)\right) s} d s\right] .
\end{aligned}
$$

The resulting model dielectric function used in this study is called the Gauss-Lorentz oscillator type. The $n$th GaussLorentz oscillator model is expressed in (8), where $\gamma_{n}(s)=$ $\Gamma_{n}+2 \sigma_{n}^{2} s(s=t / \hbar)$ is the broadening of the $n$th oscillator, $A_{n}$ is the amplitude, and $E_{n}$ is the critical point (CP) energy. The resulting dielectric function corresponds to $\tilde{\epsilon}(\omega)=$ $\sum_{n} \tilde{\epsilon}_{n}(\omega)$. This peak function is Kramers-Kronig consistent and the broadening varies smoothly between Lorentzian $\left(\Gamma_{n}\right)$ and Gaussian $\left(\sigma_{n}\right)$. In the fitting procedure, for convenience of the practical fit procedure, broadening is allowed to vary smoothly between Lorentzian $\left(\Gamma_{n}\right)$ and Gaussian $\left(\sigma_{n}\right)$. The quantities $B r_{n}$ and $B \operatorname{mix}_{n}$ are further defined through the following relations

$B r_{n}^{2}=\Gamma_{n}^{2}+\sigma_{n}^{2} ; \quad e^{B \operatorname{mix}_{n}}=\Gamma_{n} / \sigma_{n}$

The intermediate parameters $\Gamma_{n}$ and $\sigma_{n}$ indicates how much each type of broadening contributes to the broadening. That is, by changing the values of $B r_{n}$ and $B \operatorname{mix}_{n}$, the shape of each oscillator can be easily controllable in the ellipsometric software window (e.g., WVASE32(R), J.A. Woollam). In 


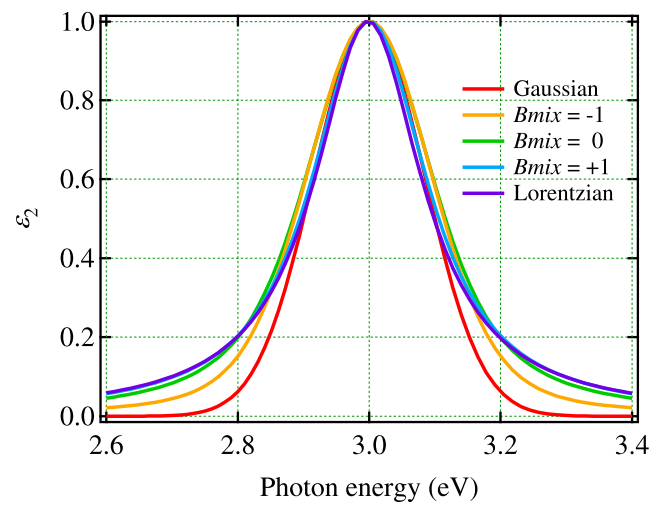

Fig. 4 Comparison of $\epsilon_{2}$ for a Gaussian, Lorentzian, as well as several Gauss-Lorentz oscillators in different cases. $A_{n}=1, E_{n}=3 \mathrm{eV}$, $B r_{n}=0.2 \mathrm{eV}$, and $B \mathrm{mix}_{n}$ is as indicated in the legend

these ways of parameterization, the shape parameter $B \operatorname{mix}_{n}$ is almost independent of the peak position, width, or area of the oscillator. When $B \operatorname{mix}_{n}$ is negative, the broadening is more Gaussian and when $B \operatorname{mix}_{n}$ is positive, the broadening is more Lorentzian from (9), as shown in Fig. 4.

\subsection{Effective medium approximation}

As briefly introduced in Sect. 3.1, in order to give more precise determination of the dielectric functions, we carried out the effective-medium approximation (EMA) in which the surface roughness layer is assumed as a physical mixture of the underlying pentacene and a void. The Bruggeman EMA used in this analysis is defined by

$f_{\mathrm{Pc}} \frac{\tilde{\epsilon}_{\mathrm{Pc}}-\tilde{\epsilon}}{\tilde{\epsilon}_{\mathrm{Pc}}+2 \tilde{\epsilon}}+f_{\text {void }} \frac{\tilde{\epsilon}_{\mathrm{void}}-\tilde{\epsilon}}{\tilde{\epsilon}_{\mathrm{void}}+2 \tilde{\epsilon}}=0$,

where $f_{\mathrm{Pc}}$ and $f_{\text {void }}$ are the volume fractions of pentacene and a void, respectively, $\tilde{\epsilon}_{\mathrm{Pc}}$ is the dielectric functions of pentacene, and $\tilde{\epsilon}$ is the resulting dielectric function of an EMA layer $\left(\tilde{\epsilon}_{\text {void }}=1\right)$ [13]. Using the relationships $f_{\text {void }}=$ $1-f_{\mathrm{Pc}}$ and $\tilde{\epsilon}_{\text {void }}=1,(10)$ is reduced to

$f_{\mathrm{Pc}} \frac{\tilde{\epsilon}_{\mathrm{Pc}}-\tilde{\epsilon}}{\tilde{\epsilon}_{\mathrm{Pc}}+2 \tilde{\epsilon}}+\left(1-f_{\mathrm{Pc}}\right) \frac{1-\tilde{\epsilon}}{1+2 \tilde{\epsilon}}=0$.

\section{Results and discussion}

\section{$4.1 \Psi$ and $\Delta$ fit}

Figure 5 shows the experimental and the calculated best-fit $\Psi$ and $\Delta$ spectra at $\phi_{0}=55^{\circ}$ and $60^{\circ}$ for thin pentacene film on $n$-Si in the spectral region of 0.7-3.1 eV. The calculated best-fit spectra were obtained using the Gauss-Lorentz model dielectric function (8) and (11) with predetermined dielectric function of bare $n$-Si substrate and the thickness

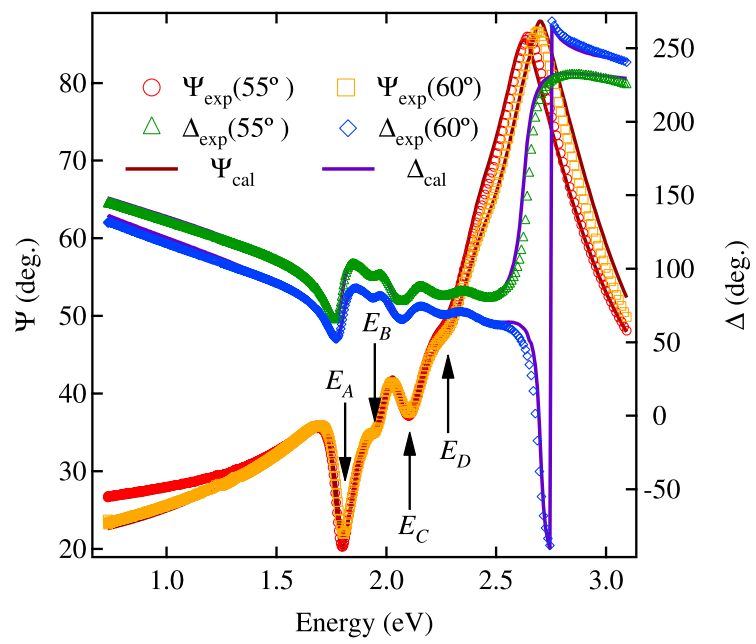

Fig. $5 \Psi$ and $\Delta$ fits of thin pentacene films on $n-\mathrm{Si}$

Table 2 Transition energies of the main features in the extinction coefficient on glass, $\mathrm{SiO}_{2}$, and $n$-Si. They are compared with the experimental measurements by Park et al. [11] and the calculation results by Tiago et al. [22]. All the values of transition energies are in $\mathrm{eV}$

\begin{tabular}{llllll}
\hline Optical transition & Glass & $\mathrm{SiO}_{2}$ & $n$-Si & $n$-Si [11] & Calc. [22] \\
\hline$E_{A}$ & 1.86 & 1.82 & 1.82 & 1.82 & 1.73 \\
$E_{B}$ & 1.96 & 1.94 & 1.94 & 1.94 & 1.86 \\
$E_{C}$ & 2.13 & 2.09 & 2.10 & 2.11 & 2.13 \\
$E_{D}$ & 2.27 & 2.26 & 2.25 & 2.25 & 2.27 \\
\hline
\end{tabular}

of each layer ( $n$-Si, pentacene film, and EMA). Arrows indicated in Fig. 5 shows the optical transitions of thin pentacene film, which are also shown in Fig. 6, tabulated in Table 2, and discussed in Sect. 4.2. With the use of a surface roughness layer and the Gauss-Lorentz model, experimental ellipsometric parameters $\Psi$ and $\Delta$ [18] were tightly fitted to model predictions with a significantly reduced mean squared error (see Fig. 5). The thickness of surface roughness layer, which can be estimated from the iterative fitting procedure, shows a good agreement with the actual roughness measured by AFM, confirming that the proposed method worked well as expected (see Table 1).

\subsection{Optical properties}

Figures 6(a) and 6(b) show the refractive indices $n$ and the extinction coefficients $k$ of thin pentacene films on glass, $\mathrm{SiO}_{2}$, and $n-\mathrm{Si}$ in the spectral region of $1-3 \mathrm{eV}$. One can easily observe that there are clear differences in the shape of the optical function measured in pentacene film deposited on different substrates. ${ }^{1}$

\footnotetext{
${ }^{1}$ The overall shape of the optical functions $n$ and $k$ of a pentacene film on $n$-Si in this work is in good agreement with that in Park et al. [11],
} 


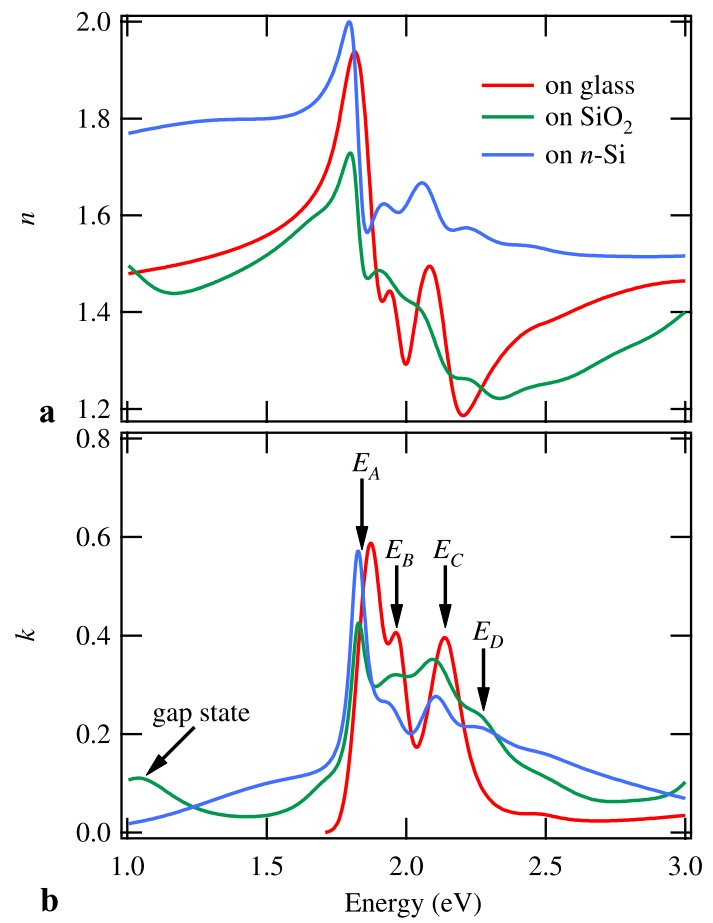

Fig. 6 Optical functions $n$ and $k$ of thin pentacene films on glass, $\mathrm{SiO}_{2}$, and $n-\mathrm{Si}$

Broad band tail, which is similar to Urbach's tail [19] in inorganic semiconductors, is seen in the extinction coefficient of a pentacene film on $\mathrm{SiO}_{2}$ and $n$-Si while it is not seen in that of a pentacene film on a glass substrate. Lang et al. [20] showed that even the purified single-crystal pentacene can have sufficient impurities to have band tails that are even broader than those observed in inorganic amorphous semiconductors. The fact that such a tail is not apparent in a film on glass may suggest that the collective interaction is less significant therein due to its relatively poor molecular ordering, while it can be more significant in films on $n-\mathrm{Si}$ and $\mathrm{SiO}_{2}$ with a higher degree of ordering.

In the extinction coefficient $k$ of a pentacene film on glass, we obtain four optical transition energies $E_{A}, E_{B}$, $E_{C}$, and $E_{D}$ with values of $1.86,1.96,2.13$, and $2.27 \mathrm{eV}$, respectively, which are in quantitative agreement with those obtained by Park et al. [11]. The first two optical transition energies, $E_{A}$ and $E_{B}$, can be assigned to $S_{1}$ excitons with a Davydov splitting of $0.1 \mathrm{eV}$ that originates from the different orientations of two pentacene atoms in a unit cell [21]. $E_{C}$ and $E_{D}$ can be regarded to be associated with the excitations to the next vibronic states of the $S_{1}$ excited state [20]. For reference, HOMO-LUMO energy gap $E_{G}$ is determined by the spectra of photoconductivity and it was previously re-

but the absolute amplitudes of the oscillators in this work are larger than those appearing therein. This is because the surface roughness is considered here to include the effect of voids. ported that $E_{G}$ of the single-crystal pentacene was around $2.2 \mathrm{eV}[19,20,22]$.

Another distinction is a small and broad peak that is found around $1 \mathrm{eV}$ in the extinction coefficient of pentacene films on $\mathrm{SiO}_{2}$. This structure appears to result from the defects formed in these films. According to deep-level transient spectroscopy measurements by Yang et al. [23], pentacene films on $\mathrm{SiO}_{2} / \mathrm{Si}$ substrates showed the energy levels of hole and electron traps located at $E_{v}+0.24 \mathrm{eV}, E_{v}+1.08$ $\mathrm{eV}, E_{v}+0.31 \mathrm{eV}$, and $E_{c}-0.69 \mathrm{eV}$. Also, this is predicted by first-principles pseudo-potential density functional calculations of the oxygen-induced gap state $\left(\mathrm{O}_{\mathrm{H}}\right.$ defect) by Northrup and Chabinyc [24], where gap state is also shown at $E \sim 1 \mathrm{eV}$. However, it is not yet clear why such a feature is not observed in the films grown on the other substrates studied.

Note that pentacene layers are deposited mostly on ITO or on PEDOT:PSS on ITO for OPV while they are deposited on $\mathrm{SiO}_{2}$ for phototransistors and on $n$-Si for photodiodes. Although the optical constants for pentacene films on ITO was not done in this study for simplicity's sake (optical constants of ITO are a subject of intense study by itself), independent study indicates that the values obtained from the one on the glass slide can be used to describe the overall shape of the optical transmission of the pentacene on ITO as well as the external quantum efficiency spectra of the corresponding OPV devices relatively well. It is regarded that such applicability stems from the similarity in degree of ordering between pentacene films on glass and those on ITO glasses. Details will be described elsewhere [25].

\section{Summary}

We have determined the optical functions of thin pentacene films prepared on glass, $\mathrm{SiO}_{2}$, and $n$-Si substrates using spectroscopic ellipsometry. Gauss-Lorentz oscillator model and effective medium approach were introduced to describe the optical transitions in pentacene films and the effect of their surface roughness. The proposed method led to an excellent fit to the experimental ellipsometric data with a low mean squared error in all the cases studied here. It was revealed that there are some quantitative differences in the optical properties of the pentacene films prepared on different substrates. Although the present study is confined to pentacene films, it can be applied to many kinds of similar organic materials, and thus we believe the present work has important implications for the design and analysis of various organic optoelectronic devices.

Acknowledgements The authors thank H. Moutinho, J. Pankow, and Z. An for their assistance in characterization of pentacene films. This work was supported in part by the US Department of Energy under Contract No. DE-AC36-99GO10337 and in part by the STC Program of the National Science Foundation under Agreement Number DMR0120967, by the Office of Naval Research. 
Open Access This article is distributed under the terms of the Creative Commons Attribution Noncommercial License which permits any noncommercial use, distribution, and reproduction in any medium, provided the original author(s) and source are credited.

\section{References}

1. C.D. Dimitrakopoulos, P.R.L. Malenfant, Adv. Mater. 14, 99 (2002)

2. T.W. Kelley, L.D. Boardman, T.D. Dunbar, D.V. Muyres, M.J. Pellerite, T.P. Smith, J. Phys. Chem. B 107, 5877 (2003)

3. O.D. Jurchescu, J. Baas, T.T.M. Palstra, Appl. Phys. Lett. 84, 3061 (2004)

4. S. Yoo, B. Domercq, B. Kippelen, Appl. Phys. Lett. 85, 5427 (2004)

5. A.C. Mayer, M.T. Lloyd, D.J. Herman, T.G. Kasen, G.G. Malliaras, Appl. Phys. Lett. 85, 6272 (2004)

6. S.S. Kim, Y.S. Choi, K. Kim, J.H. Kim, S. Im, Appl. Phys. Lett. 82, 639 (2003)

7. Y. Hu, G. Dong, C. Liu, L. Wang, Y. Qiu, Appl. Phys. Lett. 89, 072108 (2006)

8. Y.-Y. Noh, D.-Y. Kim, K. Yase, J. Appl. Phys. 98, 074505 (2005)

9. P. Peumans, A. Yakimov, S.R. Forrest, J. Appl. Phys. 93, 3693 (2003)

10. P. Peumans, A. Yakimov, S.R. Forrest, J. Appl. Phys. 95, 2938 (2004)

11. S.P. Park, S.S. Kim, J.H. Kim, C.N. Whang, S. Im, Appl. Phys. Lett. 80, 2872 (2002)
12. M. Schubert, C. Bundesmann, G. Jakopic, H. Maresch, H. Arwin, Appl. Phys. Lett. 84, 200 (2004)

13. R.M.A. Azzam, N.M. Bashara, Ellipsometry and Polarized Light, 1st edn. (North-Holland, Amsterdam, 1977)

14. A.B. Djurišić, T. Fritz, C. Leo, Opt. Commun. 183, 123 (2000)

15. A.B. Djurišić, C.Y. Kwong, W.L. Guo, Z.T. Liu, H.S. Kwok, W.K. Chan, Appl. Phys. A 77, 649 (2003)

16. C.C. Kim, J.W. Garland, H. Abad, P.M. Raccah, Phys. Rev. B 45, 11749 (1992)

17. P.Y. Yu, M. Cardona, Fundamentals of Semiconductors: Physics and Materials Properties, 2nd edn. (Springer, Berlin, 1999)

18. S.-H. Han, C. Persson, F.S. Hasoon, H.A. Al-Thani, A.M. Hermann, D.H. Levi, Phys. Rev. B 74, 085212 (2006)

19. J.I. Pankove, Optical Processes in Semiconductors, 1st edn. (Prentice-Hall, Englewood Cliffs, 1971)

20. D.V. Lang, X. Chi, T. Siegrist, A.M. Sergent, A.P. Ramirez, Phys. Rev. Lett. 93, 086802 (2004)

21. E.A. Silinsh, Organic Molecular Crystals: Their Electronic States (Springer, Berlin, 1980)

22. M.L. Tiago, J.E. Northrup, S.G. Louie, Phys. Rev. B 67, 115212 (2003)

23. Y.S. Yang, S.H. Kim, J.-I. Lee, H.Y. Chu, L.-M. Do, H. Lee, J. Oh, T. Zyung, M.K. Ryu, M.S. Jang, Appl. Phys. Lett. 80, 1595 (2002)

24. J.E. Northrup, M.L. Chabinyc, Phys. Rev. B 68, 041202(R) (2003)

25. S. Yoo, W. Potscavage Jr., B. Domercq, S.-H. Han, T.-D. Li, S. Jones, R. Szoszkiewicz, D. Levi, E. Riedo, S.R. Marder, B. Kippelen, Solid-State Electron. 51, 1367 (2007) 